979

\section{INPATIENT VERSUS OUTPATIENT SETTINGS FOR PERFORMING PERCUTANEOUS RENAL BIOPSIES}

\author{
A.S. Al-Makadma1', S.I. Al-Akash² \\ ${ }^{1}$ Pediatrics, King Fahad Medical City, Riyadh, \\ Saudi Arabia, ${ }^{2}$ Pediatrics, Drisoll Children's \\ Hospital, Corpus Christi, TX, USA
}

Background: Renal biopsy is the gold standard for a definate diagnosis of a variety of renal diseases. Adequate patient preparation and evaluation are mandatory .Though invasive, renal biopsy is generally considered a safe procedure in paediatric patients.

Patients and methods: We have retrospectively reviewed the charts of 88 consecutive patients under the age of 15 years who have undergone percutaneous renal biopsies. After adequate preparation Biopsies were performed blindly under aseptic conditions. All biopsies were performed using an automated spring-loaded core biopsy needle. In most cases, the lower pole was targeted in native kidney biopsies, and the upper of pole in transplanted kidneys.

Results: Samples from 43 native kidneys (49\%), and 45from transplanted kidneys (51\%). There were no deaths, and none of the patients with haemorrhage required nephrectomy. All complications were diagnosed within the initial 4- to 6-hour observation period following the procedure. There were only 2 $(4 \%)$ and $4(9 \%)$ inadequate samples in the inpatient and outpatient groups, respectively

Discussion: The incidence of serious complications of percutaneous renal biopsy in the literature is rare. Our overall complication rates of $8.8 \%$ and $13.9 \%$ for the inpatient and outpatient procedures, respectively.Our overall rate of complications compares favourably with the rate in literatures

Conclusion: This is a further evidence that it is safe to perform percutaneous renal biopsy in children in an outpatient setup after careful screening and that observation for 4 to 6 hours after the procedure is adequate to detect complications. However, this should only be undertaken where backup services are readily available.

\section{ETIOLOGY OF ACUTE PYELONEPHRITIS AND CORRELATION OF CLINICAL AND ANALYTICAL PARAMETERS}
A. Leitão, S. Paupério, J.G. Dinis, A. Sousa
Centro Hospitalar do Médio Ave - Unidade de Santo Tirso, Santo Tirso, Portugal

Background and aims: Urinary tract infections (UTIs) are relatively common infections in children. Findings on nuclear renal scans suggest that the vast majority of infants and young children with febrile UTIs have acute pyelonephritis (APN, upper-tract infection). Early recognition and prompt treatment of UTIs is important to prevent late sequelae, such as renal scarring, hypertension, and renal failure. When assessing the pediatric patient with UTI, one may encounter few specific symptoms. Febrile UTI should be assumed to be pyelonephritis and treated accordingly. The aims of this study are determine prevalence of APN in our department, characterize etiologic agents of APN and establish correlation between clinical and analytical parameters.

Materials and methods: We studied 72 patients with acute pyelonephritis (APN) aged 1 month to 5 years, admitted between January 2004 and December 2008. Retrospective analysis of clinical processes, including analytical examination, renal ultrasonography, DMSA Scan and cistouretography was made.

Results: 72 patients aged between 1 month and 5 years. Mean age was 11 months.

\section{Escherichia coli}

Elevated C-reactive protein, leucocytosis and neutrophilia were associated with renal damage. Vesicoureteral reflux was present in $18 \%$ cases.

Renal damage was present in $16 \%$ of patients and associated with age and recurrences of acute pyelonephritis.

Conclusion: $16 \%$ of the patients with acute pyelonephritis had renal damage. There was reflux without renal damage and renal damage without reflux. Renal damage increased with age and recurrences of acute pyelonephritis.

was the most frequently isolated agent (89.9\%), followed by Proteus mirabilis (3.6\%) and Klebsiella oxytoca. 